\title{
Synthesis and Characterization of Fused-Ring Iridapyrroles
}

\author{
John R. Bleeke, \\ Department of Chemistry, Washington University, One Brookings Drive, St. Louis, Missouri 63130 \\ Phawit Putprasert, \\ Department of Chemistry, Washington University, One Brookings Drive, St. Louis, Missouri 63130
}

Todsapon Thananatthanachon, and

Department of Chemistry, Washington University, One Brookings Drive, St. Louis, Missouri 63130

Nigam P. Rath

Department of Chemistry and Biochemistry, University of Missouri-St. Louis, One University

Boulevard, St. Louis, Missouri 63121

\section{Summary}

Treatment of aromatic nitriles with methyllithium produces $\mathrm{N}$-lithiated imine reagents which, when reacted with $\left(\eta^{2}\right.$-cyclooctene $)(\mathrm{Cl}) \operatorname{Ir}\left(\mathrm{PMe}_{3}\right)_{3}$, generate fused iridaazacycles via ortho-metallation. Mono-protonation of these iridaazacycles produces fused iridapyrrole derivatives, while diprotonation leads to several different pathways.

\begin{abstract}
Over the past 25 years, a variety of aromatic metallacycles, i.e., aromatic ring systems containing a transition metal, have been synthesized, and their physical and chemical properties have been investigated.1 Our group has focused on synthesizing a family of iridium-containing aromatic ring systems, which currently includes iridabenzene, iridapyrylium, iridathiabenzene, iridafuran, and iridathiophene. ${ }^{2}$ All of these compounds have been produced by reacting " $(\mathrm{Cl})$ $\mathrm{Ir}\left(\mathrm{PR}_{3}\right)_{3}$ " with pentadienide or heteropentadienide reagents and using iridium-centered $\mathrm{C}-\mathrm{H}$ bond activation to construct the six- or five-membered rings. Further manipulations then create the aromatic ring systems. Conspicuous from the above list of aromatic iridacycles are the nitrogen-containing systems, iridapyrrole ${ }^{3}$ and iridapyridine. ${ }^{4}$ To date, we have not succeeded in using azapentadienide reagents to construct these desired nitrogen-containing iridacycles. ${ }^{5}$ However, in this communication, we report a new approach to the synthesis of fused fivemembered iridaazacycles and their conversion to fused iridapyrroles.
\end{abstract}

As shown in Scheme 1, treatment of benzonitrile with methyllithium in THF produces Nlithiated imine reagent $\mathbf{A}$ in situ. ${ }^{6}$ Further treatment with $\left(\eta^{2}\right.$-cyclooctene $)(\mathrm{Cl}) \operatorname{Ir}\left(\mathrm{PMe}_{3}\right)_{3}, 7$ followed by stirring for 30 minutes, produces the fused iridaazacycle, fac-1, in $90 \%$ isolated yield. The likely intermediate in this reaction is $16 \mathrm{e}^{-}$species $\mathbf{B}$ which undergoes iridiumcentered $\mathrm{C}-\mathrm{H}$ bond activation at the ortho position of the phenyl ring. The fac geometry of product 1 is evident from the ${ }^{31} \mathrm{P}\left\{{ }^{1} \mathrm{H}\right\}$ NMR spectrum, which exhibits three distinct phosphorus signals for the three different phosphine ligands. ${ }^{8}$ In the ${ }^{1} \mathrm{H}$ NMR, the four phenyl ring protons appear in the region $\delta 7.19-7.70$, while the methyl resonates at $\delta 2.61$. The metal-hydride is observed at $\delta-10.87$ and exhibits a large coupling $(137.0 \mathrm{~Hz})$ due to the trans phosphine and

Correspondence to: John R. Bleeke.

Supporting Information Available: Detailed syntheses and characterization of compounds 1-9; structure determination summaries and listings of final atomic coordinates, thermal parameters, bond lengths, bond angles, and torsional angles for compounds fac-2 (triflate salt), fac-4 (triflate salt), fac-6 (chloride salt), and $\mathbf{m e r}-\mathbf{9}$ (triflate salt). This material is available free of charge via the Internet at http://pubs.acs.org. 
small couplings $(\sim 20 \mathrm{~Hz})$ due to the cis phosphines. In the ${ }^{13} \mathrm{C}\left\{{ }^{1} \mathrm{H}\right\} \mathrm{NMR}, \mathrm{C} 1$ resonates farthest downfield at $\delta 173.0$, while the phenyl ring carbons appear in the region $\delta 120.7-158.0$. Significantly, the signal for C3 (at $\delta 158.0$ ) is a widely-spaced doublet $\left(\mathrm{J}_{\mathrm{C}-\mathrm{P}}=81.6 \mathrm{~Hz}\right.$ ), indicating that it is bound to iridium and strongly coupled to a trans phosphine. The methyl group resonates at $\delta 25.7$.

Treatment of $f a c-1$ with one equivalent of triflic acid in THF leads to protonation at the nitrogen center and production of the iridaisoindole, $\boldsymbol{f a c}-\mathbf{2}$ (Scheme 1), in high yield. The NMR spectra of $\boldsymbol{f a c} \mathbf{- 2}$ are very similar to those of $\boldsymbol{f a c}-\mathbf{1}$, except for the appearance of a new broad singlet at $\delta 9.70$, attributable to the proton on nitrogen. ${ }^{9}$ This new signal shows a strong correlation with the ring methyl signal in the ${ }^{1} \mathrm{H}$ COSY NMR spectrum. The detailed NMR spectra of $f a c-2$ and the other compounds reported herein are available in Supporting Material.

The X-ray crystal structure of $\boldsymbol{f a c} \mathbf{- 2}$ has been obtained and is shown in Figure 1, while key bond distances are summarized in the figure caption. The 5,6 fused ring system is highly planar with a mean deviation of only $0.043 \AA$ A Two reasonable resonance structures, I and II (Drawing 1), can be proposed for iridaisoindole 2. Both structures appear to contribute to the bonding, as evidenced by the partial delocalization of carbon-carbon bonding within the five-membered ring $(\mathrm{C} 1-\mathrm{C} 2=1.457(4) \AA ; \mathrm{C} 2-\mathrm{C} 3=1.423(4) \AA)$. However, the relatively short $\mathrm{C} 1-\mathrm{N} 1$ distance (1.280(4) $\AA$ ), along with the relatively long C1-C2 distance, suggests that II is the more important of the two contributors, perhaps because it includes a fully conjugated (aromatic) six-membered ring. In this context, it is interesting to note that organic isoindoles readily undergo tautomerization to isoindolenine structures (Drawing 2), a process which restores full conjugation to the carbocyclic ring. ${ }^{10}$

As shown in Scheme 2, the same methodology can be used to generate related fused ring systems. Hence, treatment of 2-cyanopyridine with methyllithium, followed by $\left(\eta^{2}-\right.$ cyclooctene $)(\mathrm{Cl}) \operatorname{Ir}\left(\mathrm{PMe}_{3}\right)_{3},{ }^{7}$ produces the 5,6 fused ring system, fac $-\mathbf{3}$, via ortho $\mathrm{C}-\mathrm{H}$ bond activation (Scheme 2, top). In the ${ }^{1} \mathrm{H}$ NMR spectrum of $\mathbf{f a c}-\mathbf{3}$, the three pyridyl protons resonate in the region of $\delta 6.86-8.57$, while the ring methyl appears at $\delta 3.04$. The metal-hydride resonates at $\delta-11.00$ and, as expected, is split into an apparent doublet of triplets with one very large coupling $(134.1 \mathrm{~Hz})$, due to the trans phosphine, and two smaller couplings $(21.0 \mathrm{~Hz})$ due to the cis phosphines. In the ${ }^{13} \mathrm{C}\left\{{ }^{1} \mathrm{H}\right\}$ NMR spectrum, the signal for $\mathrm{C} 1$ is the most downfield at $\delta$ 175.4, while the pyridyl carbons resonate between $\delta 121.3$ and $\delta 173.5$. The signal for $\mathrm{C} 3$ is split into a widely-spaced doublet $\left(\mathrm{J}_{\mathrm{C}-\mathrm{P}}=86.0 \mathrm{~Hz}\right)$, indicating that it is bonded to iridium and located trans to a phosphine. Treatment of $f a c-3$ with one equivalent of triflic acid leads to protonation at the nitrogen of the five-membered ring and production of the iridapyrrole derivative, $f a c-4$. The NMR spectra of $f a c-4$ strongly resemble those of $f a c-3$, except for the addition of a broad NH resonance at $\delta 10.45$. The structure of $f a c-4$ has been confirmed by X-ray crystallography and is presented in Figure 2. The key bond distances, summarized in the figure caption, are very similar to those of $\mathbf{f a c - 2}$, suggesting that resonance structure IV (Drawing 3) is the more important contributor to the bonding, probably because it includes a fully conjugated pyridine ring.

Using the same synthetic approach, treatment of 2-thiophenecarbonitrile with methyllithium, followed by $\left(\eta^{2}\right.$-cyclooctene $)(\mathrm{Cl}) \operatorname{Ir}\left(\mathrm{PMe}_{3}\right)_{3},{ }^{7}$ produces the 5,5 fused ring system, $\boldsymbol{f a c}$-5, via ortho $\mathrm{C}-\mathrm{H}$ bond activation (Scheme 2, bottom). Treatment of fac $\mathbf{5}$ with one equivalent of triflic acid again leads to protonation at nitrogen and production of iridapyrrole derivative fac-6. As with iridapyrroles fac-2 and fac-4, one can draw two reasonable resonance structures for fac-6 (Drawing 4). However, in this case we predict that resonance structure $\mathbf{V}$ (Drawing 4) will be a more significant contributor than are I or III (vide supra) because the alternative structure, VI, has less to gain by fully conjugating the fused thiophene ring. ${ }^{11}$ This prediction is, in fact, supported by the X-ray crystal structure of $\boldsymbol{f a c}-\mathbf{6}$, which has been obtained for the 
chloride salt, and is presented in Figure 3. As reported in the figure caption, the carbon-carbon bond lengths within the five-membered iridapyrrole ring of $\mathbf{f a c}-\mathbf{6}$ are virtually identical (C1$\mathrm{C} 2=1.394(10) \AA$; $\mathrm{C} 2-\mathrm{C} 3=1.396(11) \AA$ ), implying that resonance structures $\mathbf{V}$ and $\mathbf{V I}$ contribute equally to the bonding.

As shown in Scheme 3, each of the fused iridapyrrole complexes (fac-2,fac-4, and fac-6) is reactive toward a second equivalent of triflic acid. Interestingly, three different kinds of reaction products are observed. Treatment of $\boldsymbol{f a c}-\mathbf{2}$ with triflic acid leads to formation of $m e r-7$, a close analogue of $f a c-2$, in which triflate has replaced hydride in the metal's coordination sphere. A likely mechanism for this reaction involves protonation at iridium, followed by reductive elimination of $\mathrm{H}_{2}$ and triflate attack on the resulting $16 \mathrm{e}^{-}$dicationic iridium center. The mer geometry of 7 is evident from the ${ }^{31} \mathrm{P}\left\{{ }^{1} \mathrm{H}\right\}$ NMR spectrum, which consists of just two signals, a doublet and a triplet in a characteristic 2:1 ratio. The ${ }^{1} \mathrm{H}$ NMR spectrum of $\boldsymbol{m e r}-\mathbf{7}$ is similar to that of $\boldsymbol{f a c}-\mathbf{2}$ except for the absence of a metal-hydride signal. In the ${ }^{13} \mathrm{C}\left\{{ }^{1} \mathrm{H}\right\}$ NMR spectrum of $\boldsymbol{m e r}-7$, the signal for $\mathrm{C} 3$ is no longer coupled strongly to phosphorus, indicating that the triflate ligand (not $\mathrm{PMe}_{3}$ ) must reside trans to $\mathrm{C} 3$, as drawn in Scheme 3.

In contrast, treatment of $\mathbf{f a c}-\mathbf{4}$ with a second equivalent of triflic acid results in simple protonation at the pyridine nitrogen and production of $f a c-8$ (Scheme 3, middle). The ${ }^{1} \mathrm{H}$ NMR spectrum of $\boldsymbol{f a c} \mathbf{- 8}$ is similar to that of $\boldsymbol{f a c} \mathbf{- 4}$, except for the appearance of a new broad NH signal at $\delta 14.53$, which we have assigned to the pyridinium proton. ${ }^{12}$ The iridapyrrole proton appears at $\delta 11.17$, slightly downfield from its position at $\delta 10.45$ in precursor $f a c-4$. The assignments of these protons are confirmed by the ${ }^{1} \mathrm{H}$ COSY NMR spectrum; the signal at $\delta$ 14.53 correlates with the signal for pyridine ring proton H6, while the signal at $\delta 11.17$ correlates with the ring methyl group.

Finally, treatment of $\mathbf{f a c}-\mathbf{6}$ with a second equivalent of triflic acid results in skeletal rearrangement of the fused ring system and production of mer-9 (Scheme 3, bottom) in which sulfur is now bonded to iridium. Like the reaction of $\boldsymbol{f a c}$-2 described above, this reaction probably involves initial protonation at iridium. But instead of reductive elimination of $\mathrm{H}_{2}$, the thiophene ring $(\mathrm{C} 3-\mathrm{H})$ eliminates, rotates, and then recoordinates through sulfur. The driving force for this rearrangement is probably relief of strain within the planar 5,5 fused ring system.

The structure of $\boldsymbol{m e r - 9}$ has been confirmed by X-ray crystallography and is shown in Figure 4. The ring system is highly non-planar as a result of sulfur's rehybridization to $\mathrm{sp}^{3}$. The dihedral angle between the thiophene ring and the molecule's equatorial plane (Ir $1 / \mathrm{P} 1 / \mathrm{S} 1 / \mathrm{N} 1)$ is $40.9^{\circ}$. As expected, bonding within the ring system is rather localized (see caption to Figure 4). While the solid-state structure of $\boldsymbol{m e r - 9}$ is highly non-planar, it displays mirror plane symmetry in solution by NMR. Hence, the two axial phosphines are equivalent by room temperature ${ }^{31} \mathrm{P}$ $\left\{{ }^{1} \mathrm{H}\right\}$ NMR, implying that inversion at sulfur is facile. ${ }^{13}$ All other features of the NMR are fully consistent with the X-ray structure.

In this communication, we have introduced a new approach to the synthesis of five-membered iridaazacycles, using aromatic nitriles as the organic building blocks and $\mathrm{CH}$ bond activation to close the rings. Mono-protonation of these iridaazacycles leads to the production of novel iridapyrrole derivatives containing fused rings. Di-protonation, on the other hand, results in a variety of reaction pathways, only some of which leave the fused iridapyrrole framework intact.

\section{Supplementary Material}

Refer to Web version on PubMed Central for supplementary material. 


\section{Acknowledgments}

P.P was supported by a Thai Government Scholarship. Washington University's High-Resolution NMR Service Facility was funded in part by NIH Support Instrument Grants (RR-02004, RR-05018, and RR-07155). The regional X-ray Facility at the University of Missouri-St. Louis was funded in part by the National Science Foundation's MRI Program (CHE-0420497).

\section{References and Notes}

1. Recent reviews: a) Bleeke JR. Chem. Rev 2001;101:1205-1227. [PubMed: 11710218] b) He G, Xia H, Jia G. Chin. Sci. Bull 2004;49:1543-1553. c) Wright LJ. J. Chem. Soc., Dalton Trans 2006:18211827. d) Landorf CW, Haley MM. Angew. Chem. Int. Ed 2006;45:3914-3936.

2. Bleeke JR. Acc. Chem. Res 2007;40:1035-1047. [PubMed: 17595050]

3. Several examples of iridapyrroles have been obtained using a 3+2 cycloaddition route: a) Alvarado Y, Daff PJ, Pérez PJ, Poveda ML, Sáchez-Delgado R, Carmona E. Organometallics 1996;15:2192-2194. b) Alias FM, Daff PJ, Paneque M, Poveda M, Carmona E, Pérez PJ, Salazar V, Alvarado Y, Atencio R, Sánchez-Delgado R. Chem. Eur. J 2002;8:5132-5146.

4. Iridapyridines are unknown. In fact, only one example of a metallapyridine, a tantalum derivative, has been reported: Weller KJ, Filippov I, Briggs PM, Wigley DE. Organometallics 1998;17:322.

5. Reactions involving the bulky t-butylazapentadienide reagent result in the formation of allyl-iridium products: Bleeke JR, Luaders ST, Robinson KD. Organometallics 1994;13:1592-1600.

6. Similar N-metallated imines have been produced by Erker: Erker G, Riedel M, Koch S, Jödicke T, Würthwein E-U. J. Org. Chem 1995;60:5284-5290.

7. Herskovitz T, Guggenberger LJ. J. Am. Chem. Soc 1976;98:1615-1616. In solution, this species dissociates cyclooctene to produce the reactive $16 \mathrm{e}^{-}$"(Cl) $\operatorname{Ir}\left(\mathrm{PMe}_{3}\right)_{3} "$.

8. Over time, fac-1 slowly converts to its mer isomer, $\boldsymbol{m e r}-\mathbf{1}$, a reaction that can be easily monitored by ${ }^{31} \mathrm{P}$ NMR spectroscopy. Slow fac to $m e r$ isomerizations are also observed for analogues $\mathbf{3}$ and $\mathbf{5}$ (vide infra).

9. For organic pyrroles, the NH signal is typically a broad resonance in the region $\delta 7-\delta 12$ : Pouchert CJ, Behnke J. The Aldrich Library of ${ }^{13} \mathrm{C}$ and ${ }^{1}$ H FT NMR Spectra: Aldrich Chemical Company 1993; Vol. III:1-13.

10. a) Bird, CW.; Cheeseman, GWH. Comprehensive Heterocyclic Chemistry. Katritzky, AR.; Rees, CW., editors. Vol. Vol. 4. Oxford: Pergamon Press; 1984. p. 1-38.b) Carey, FA.; Sundberg, R. Advanced Organic Chemistry. 4th Edition. New York: Kluwer Academic/Plenum Publishers; 2000. p. 540-543.

11. By a variety of criteria, thiophene is judged to be less aromatic than benzene or pyridine. See: Bird CW. Tetrahedron 1996;52:9945-9952.

12. For organic pyridiniums, the NH signal is typically very broad and very downfield (often downfield from $\delta 15)$ : Pouchert, CJ.; Behnke, J. The Aldrich Library of ${ }^{13} \mathrm{C}$ and ${ }^{1} \mathrm{H}$ FT NMR Spectra. Vol. Vol. III. Aldrich Chemical Company; p. 237

13. While the activation energy is rather low for inversion about sulfonium sulfur, the planar intermediate would be stabilized by overlap of the filled sulfur $\mathrm{p} \pi$ orbital with the ring's carbon $\pi$-system: Anderson, KK. The Chemistry of the Sulphonium Group. Stirling, CJM., editor. Vol. 1981. England: Wiley: Chichester; 1981. p. 229-266. 


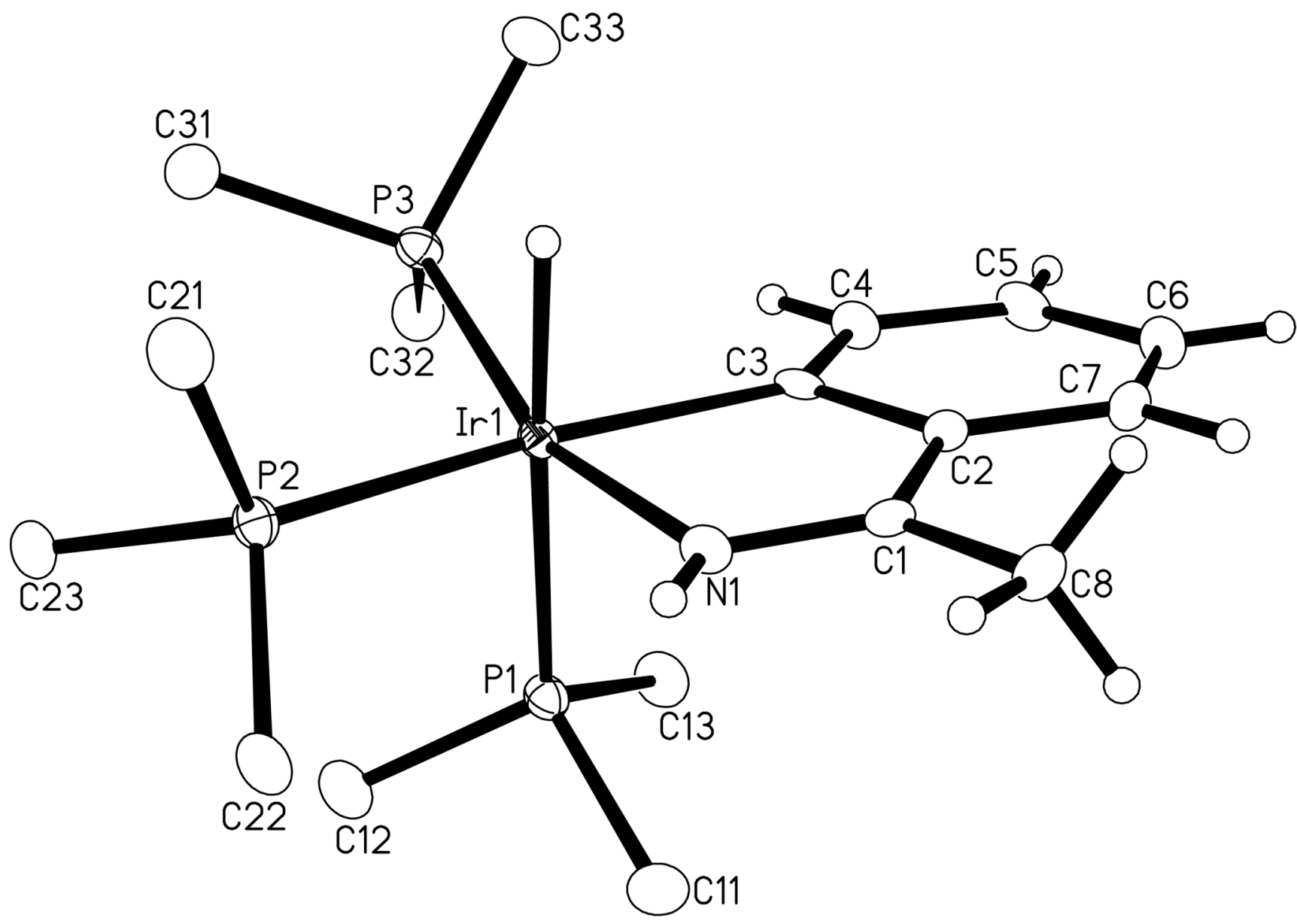

Figure 1.

Molecular structure of cation fac-2, using thermal ellipsoids at the 50\% level. $\mathrm{PMe}_{3}$ methyl H's and triflate anion are not shown. Selected bond distances ( $)$ : Ir1-P1, 2.3600(8); Ir1-P2, 2.3488(8); Ir1-P3, 2.2711(8); Ir1-N1, 2.096(3); Ir1-C3, 2.084(3); Ir1-H1, 1.62(3) N1-C1, 1.280 (4); C1-C8, 1.499(4); C1-C2, 1.457(4); C2-C3, 1.423(4); C3-C4, 1.403(4); C4-C5, 1.383(4); C5-C6; 1.389(4); C6-C7, 1.376(4); C2-C7, 1.396(4). 


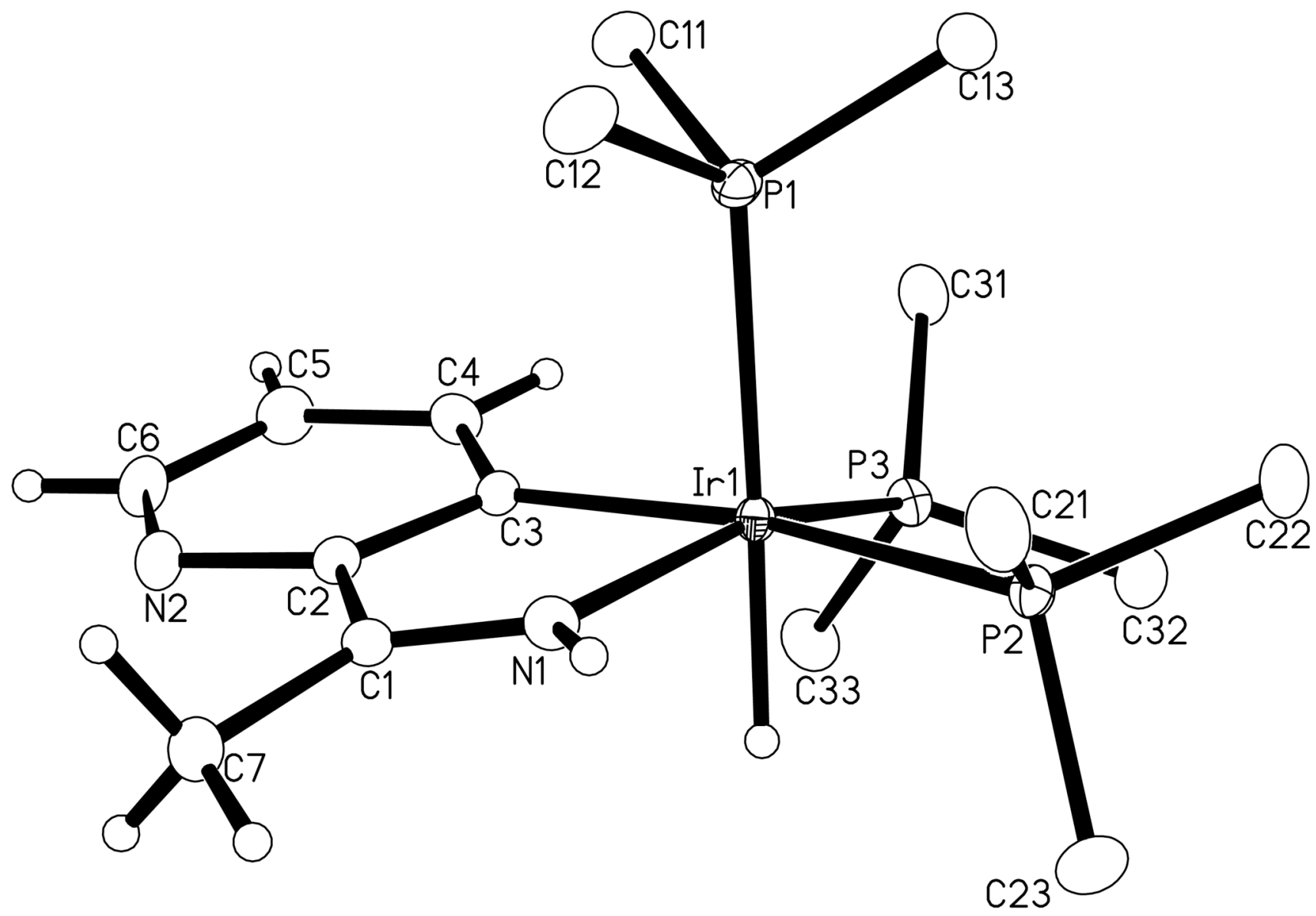

Figure 2.

Molecular structure of cation fac-4, using thermal ellipsoids at the 50\% level. $\mathrm{PMe}_{3}$ methyl H's and triflate anion are not shown. Selected bond distances ( $\AA$ ): Ir1-P1, 2.3590(7); Ir1-P2, 2.3453(7); Ir1-P3, 2.2670(7); Ir1-N1, 2.101(2); Ir1-C3, 2.083(2); Ir1-H1, 1.59(3) N1-C1, 1.285 (3); C1-C7, 1.491(4); C1-C2, 1.460(4); C2-C3, 1.415(4); C3-C4, 1.402(4); C4-C5, 1.385(4); C5-C6; 1.389(4); C6-N2, 1.328(4); C2-N2, 1.350(3). 


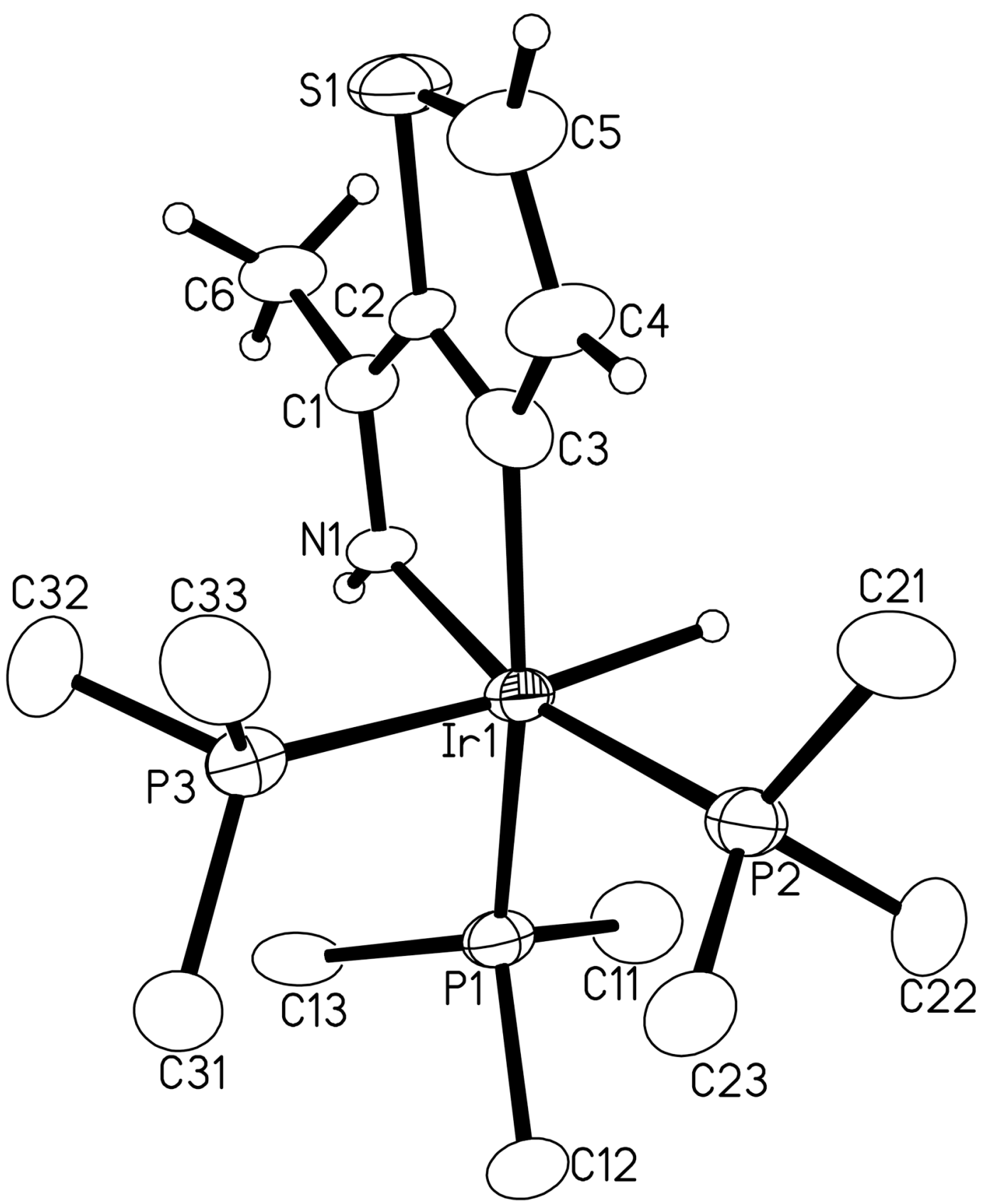

Figure 3.

Molecular structure of cation $\mathbf{f a c}-\mathbf{6}$, using thermal ellipsoids at the $50 \%$ level. $\mathrm{PMe}_{3}$ methyl H's, chloride anion, and toluene solvent molecule are not shown. Selected bond distances $(\AA)$ : Ir1-P1, 2.333(2); Ir1-P2, 2.264(2); Ir1-P3, 2.350(2); Ir1-N1, 2.115(6); Ir1-C3, 2.101(9); Ir1H1, 1.73(6); N1-C1, 1.294(9); C1-C6, 1.514(10); C1-C2, 1.394(10); C2-C3, 1.396(11); C3C4, 1.436(11); C4-C5, 1.345(12); C5-S1, 1.696(9); C2-S1, 1.744(8). 


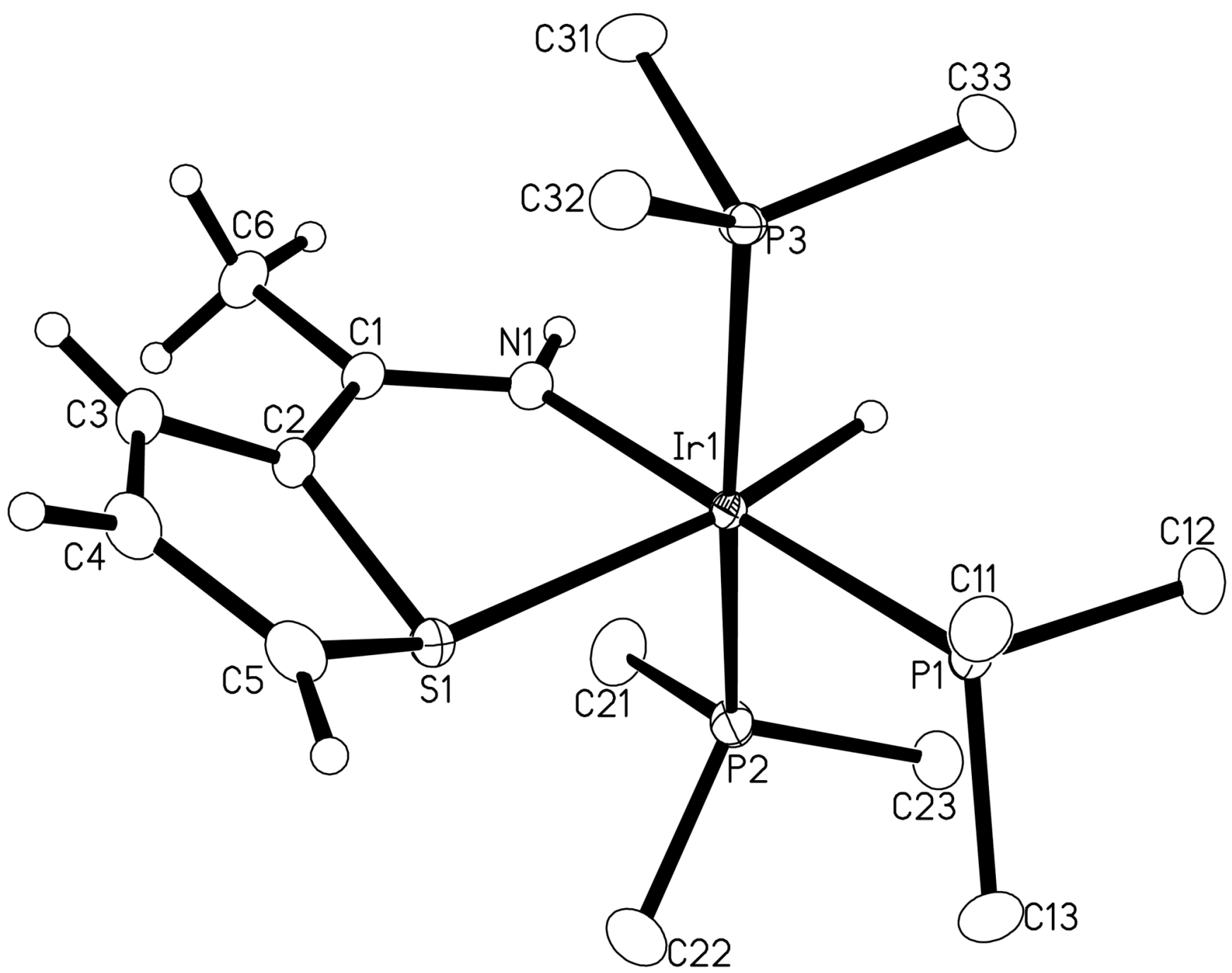

Figure 4.

Molecular structure of dication mer-9, using thermal ellipsoids at the 50\% level. $\mathrm{PMe}_{3}$ methyl H's and triflate anions are not shown. Selected bond distances $(\AA)$ : Ir1-P1, 2.2995(4); Ir1-P2, 2.3452(4); Ir1-P3, 2.3471(4); Ir1-N1, 2.1018(11); Ir1-S1, 2.4836(3); Ir1-H1, 1.55(2); N1-C1, 1.2859(17); C1-C6, 1.4894(19); C1-C2, 1.4481(19); C2-C3, 1.3610(19); C3-C4, 1.428(2); C4C5, 1.358(2); C5-S1, 1.7295(14); C2-S1, 1.7393(14). 


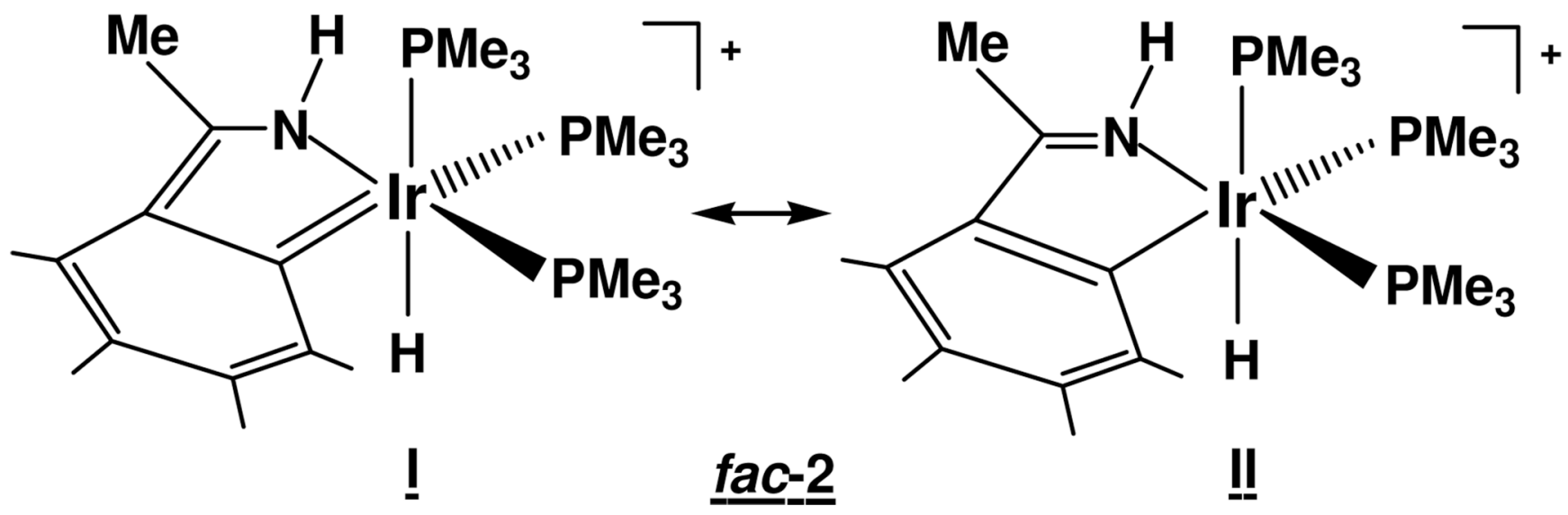

Drawing 1. 


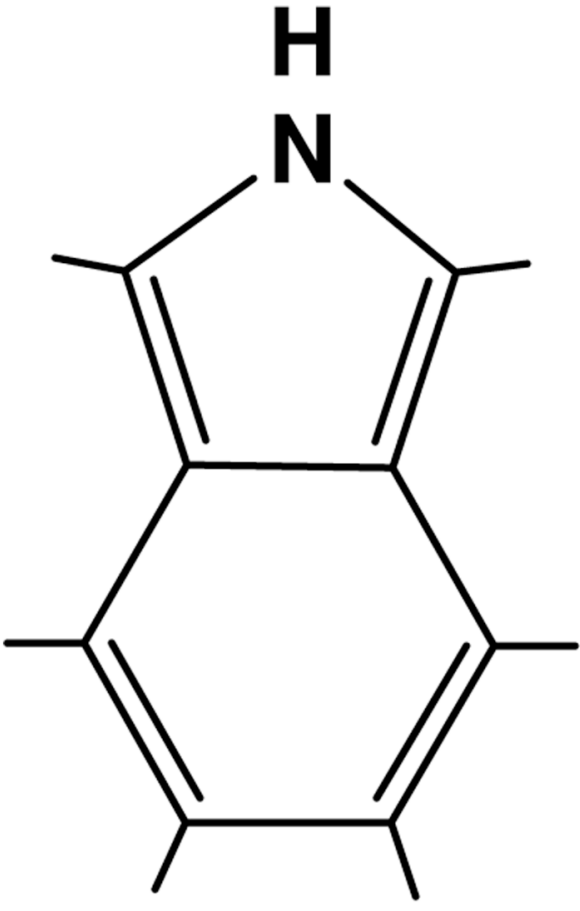

isoindole

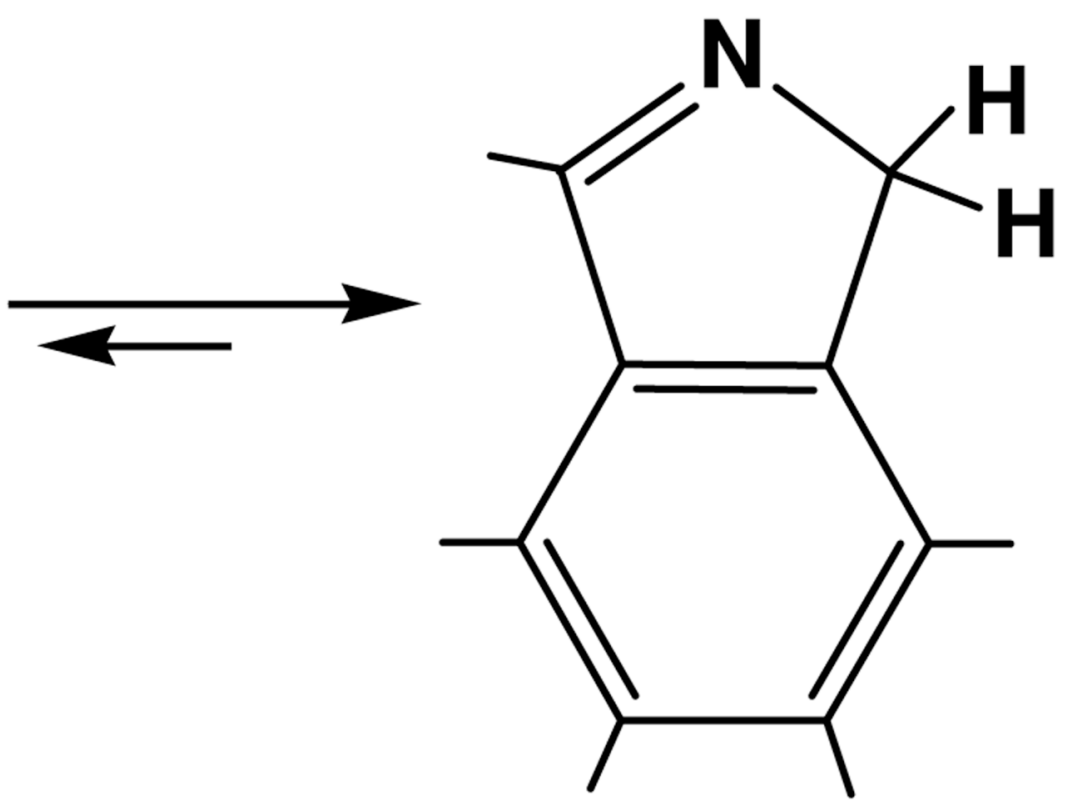

isoindolenine

Drawing 2. 

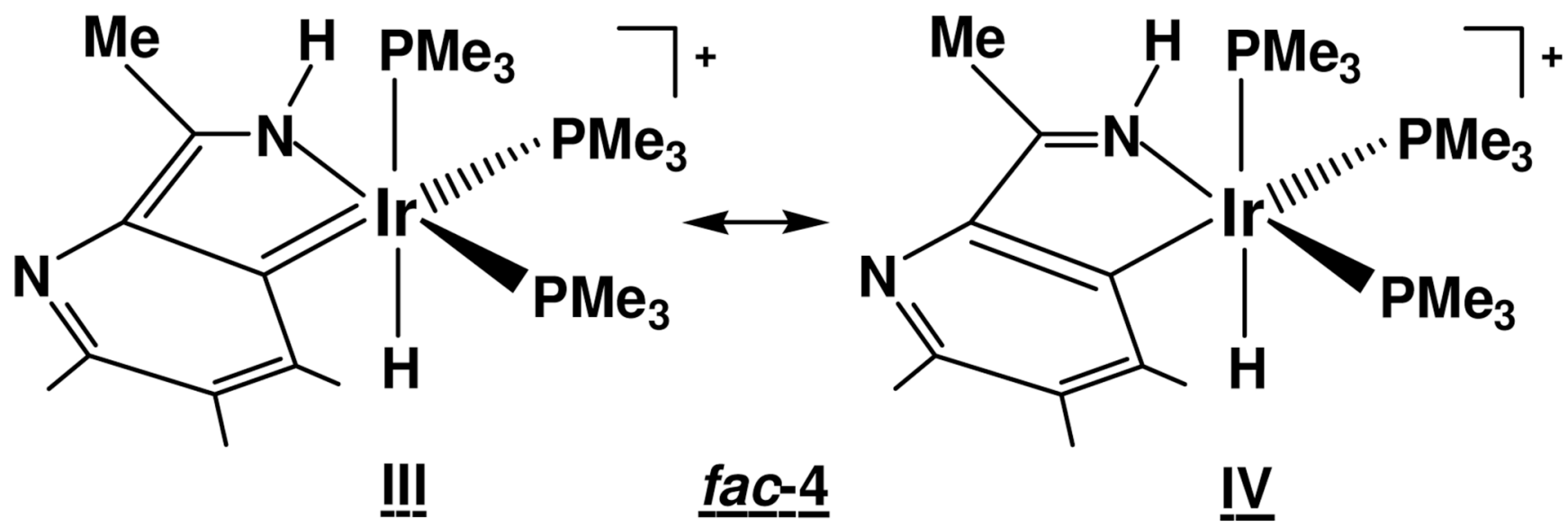

Drawing 3. 


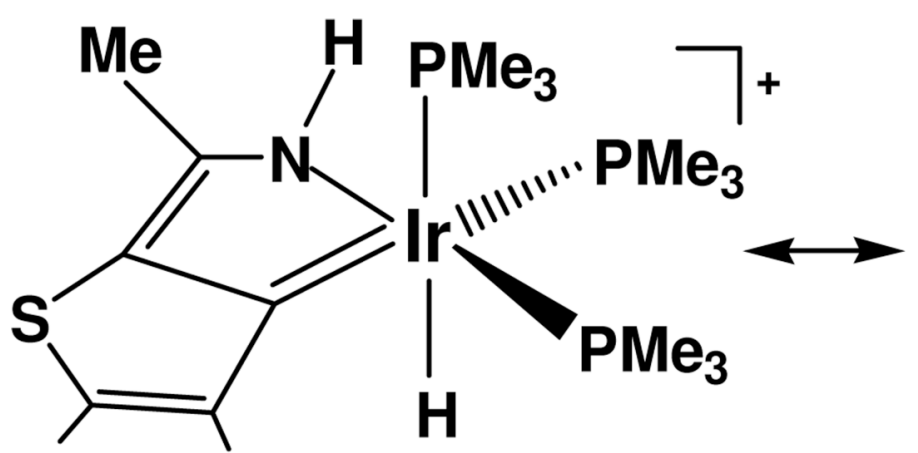

V

$\underline{f a c}-\underline{6}$

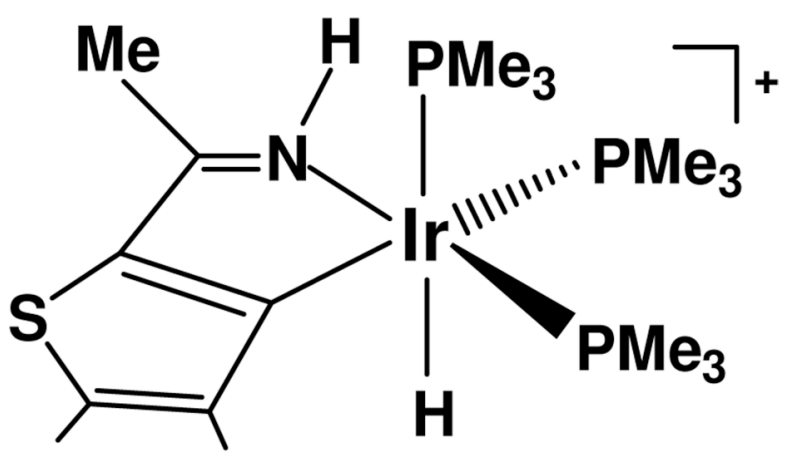

VI

Drawing 4. 

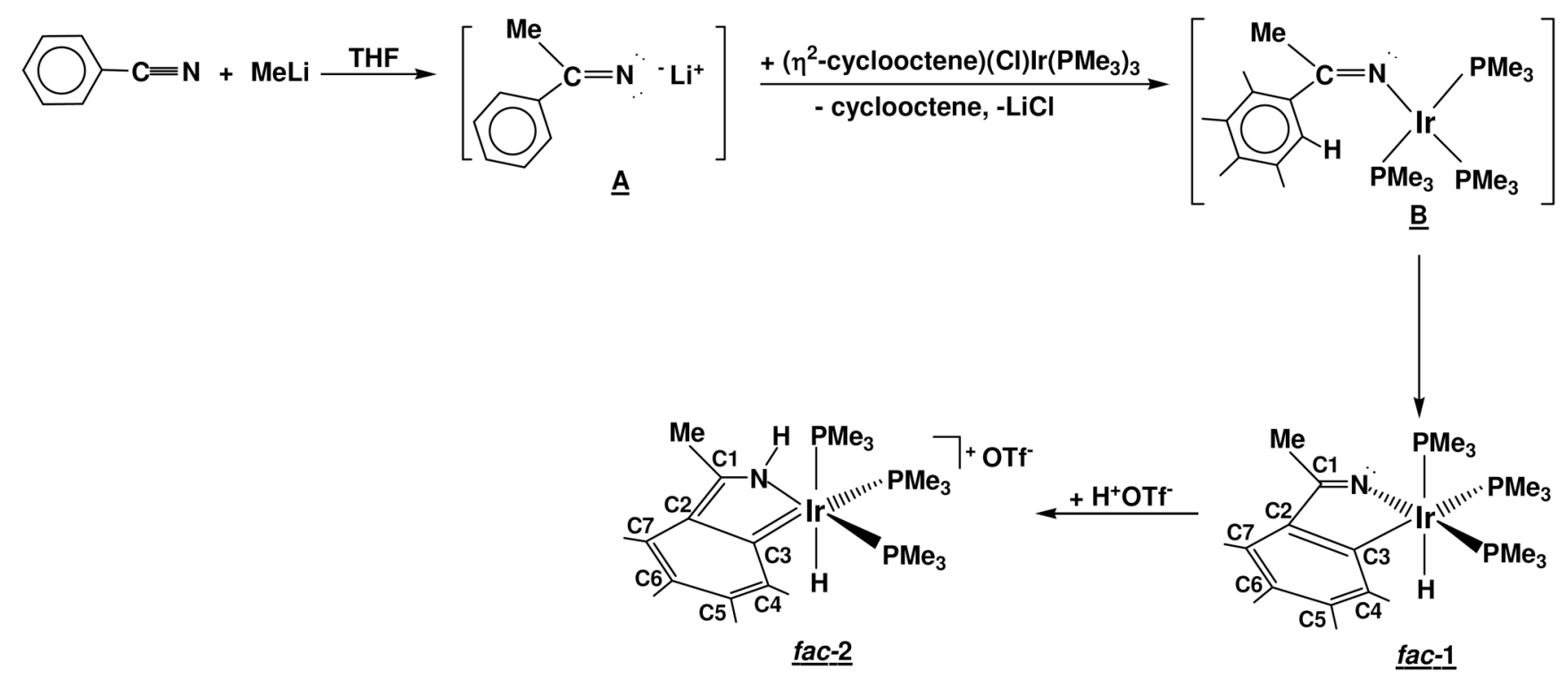

Scheme 1. 


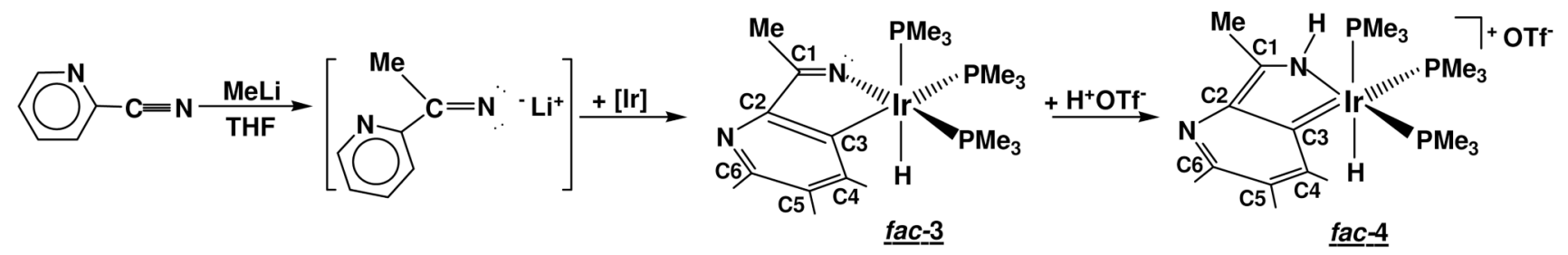

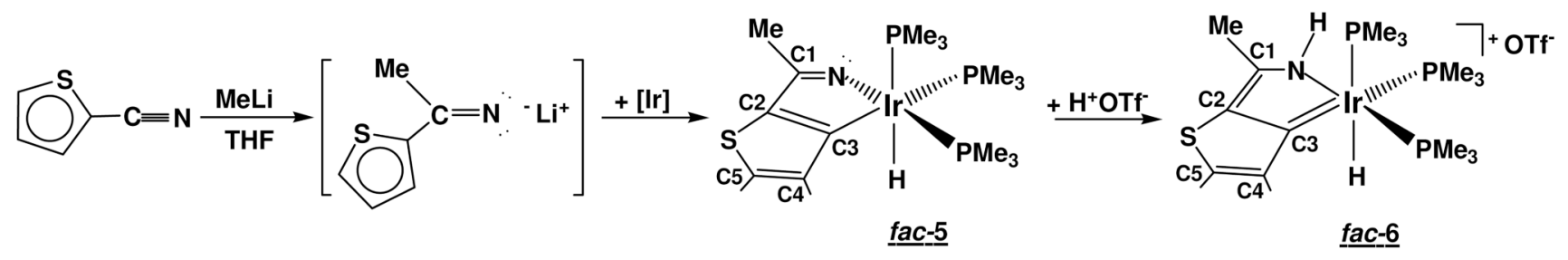

$[\mathrm{Ir}]=\left(\eta^{2}\right.$-cyclooctene $)(\mathrm{Cl}) \operatorname{Ir}\left(\mathrm{PMe}_{3}\right)_{3}$

Scheme 2. 


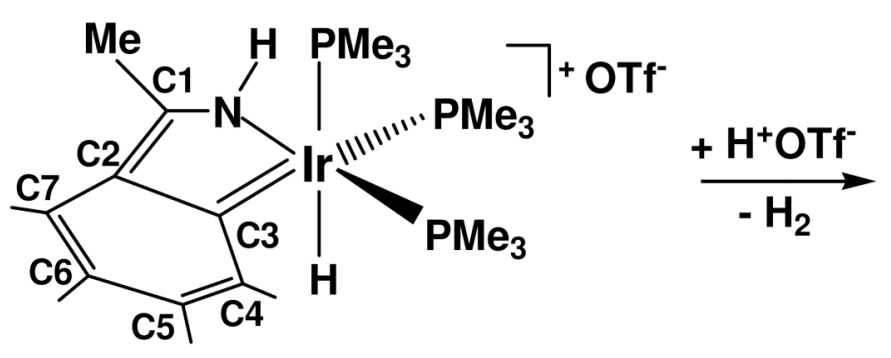

$\underline{f a c}-2$

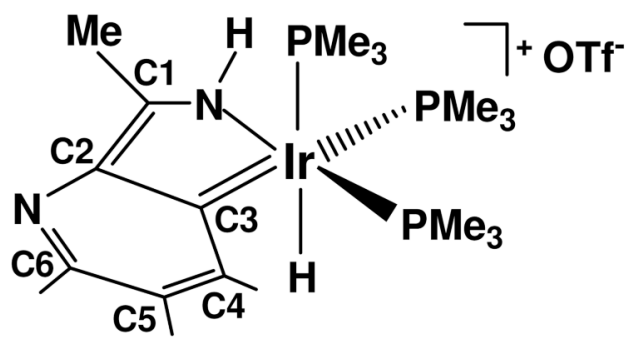

fac- 4

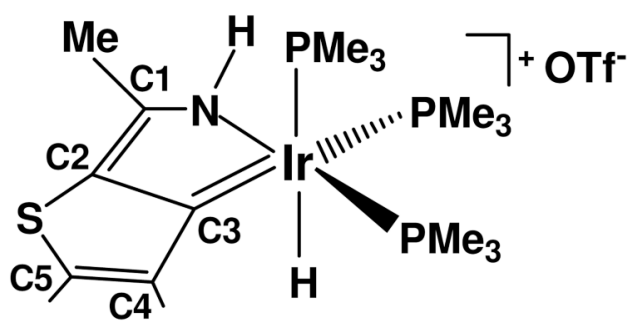

fac- $\underline{6}$

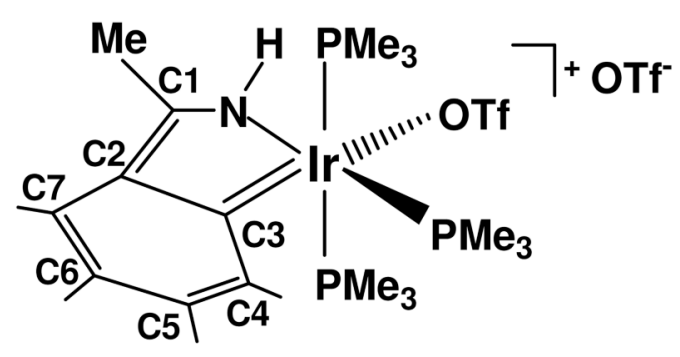

mer-7

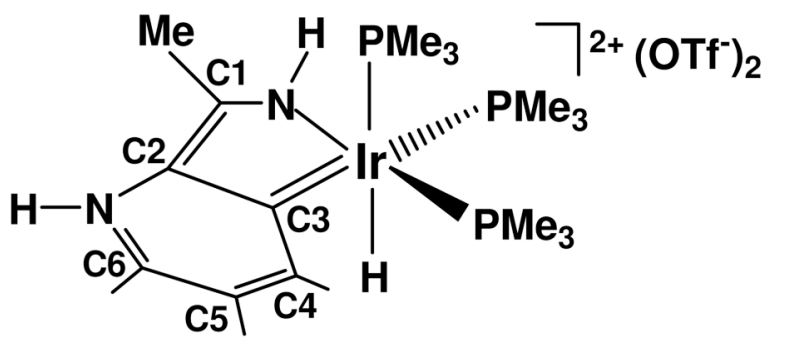

$\underline{f a c-8}$

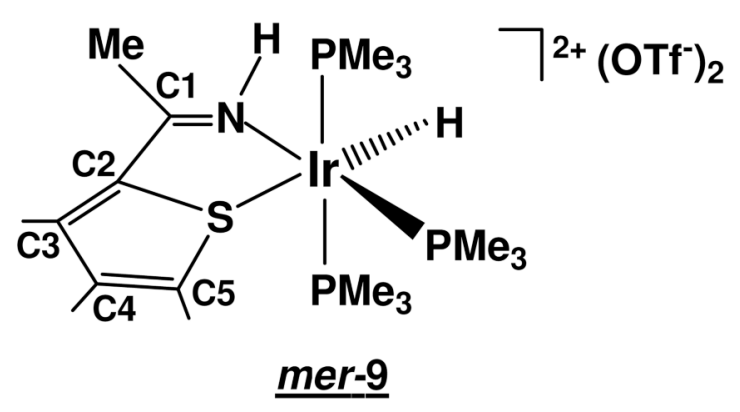

Scheme 3. 